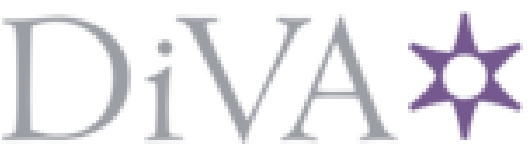

http://www.diva-portal.org

Preprint

This is the submitted version of a paper presented at 2017 IEEE International Geoscience and Remote Sensing Symposium (IGARSS).

Citation for the original published paper:

Sievert, T. (2017)

Determining the refractivity at the bottom of the atmosphere using radio occultation. In: 2017 IEEE International Geoscience and Remote Sensing Symposium (IGARSS) (pp. 4433-4436).

N.B. When citing this work, cite the original published paper.

Permanent link to this version:

http://urn.kb.se/resolve?urn=urn:nbn:se:bth-15745 


\title{
DETERMINING THE REFRACTIVITY AT THE BOTTOM OF THE ATMOSPHERE USING RADIO OCCULTATION
}

\author{
Thomas Sievert ${ }^{1}$, Joel Rasch ${ }^{2}$, Anders Carlström ${ }^{3}$, Mats I. Pettersson ${ }^{1}$, Viet Vu ${ }^{1}$ \\ ${ }^{1}$ Blekinge Institute of Technology, Karlskrona, Sweden \\ ${ }^{2}$ Molflow, Gothenburg, Sweden \\ ${ }^{3}$ RUAG Space AB, Gothenburg, Sweden
}

\begin{abstract}
High accuracy of impact height is important to get reliable Radio Occultation ( $\mathrm{RO}$ ) measurements of the atmosphere refractivity. We have made an investigation on how accurately we can measure the impact height at ground level using wave optics simulations, realistic refractivity profiles, a realistic simulator for an advanced RO instrument including noise, and using phase matching for the inversion. The idea of the investigation is to increase the measurement accuracy of impact height at low altitudes and to give reliable measurements even in cases of super-refractive layers. We present statistics on the accuracy and precision of the determination of the impact height at ground, as well as the resulting accuracy and precision in the measured refractivity.
\end{abstract}

Index Terms - radio occultation, GPS, marine boundary layer

\section{INTRODUCTION}

The radio occultation ( $\mathrm{RO}$ ) technique is a method for sounding the Earth's atmosphere by inverting global navigation satellite system (GNSS) signals that pass through it without hitting the ground. By applying the inverse Abel transform the bending angles of the optical rays can be found. From the found bending angles, it is possible to retrieve the atmosphere's refractive index, which yields valuable information about humidity, temperature and pressure at a high vertical resolution[1]. This data is mainly used in numerical weather prediction and climate research. The existence of superrefractive (SR) layers in the atmosphere makes RO measurements unreliable, as the inverted refractivity becomes negatively biased due to atmospheric ducting[2]. An approach to this problem is proposed by Xie et al. in [3]. The idea is to reconstruct SR profiles with the help of two constraints: the height corresponding to the upper boundary of the SR layer, and the impact parameter corresponding to the Earth's surface (which we refer to as $a_{\min }$ ). Xie's reconstruction is promising, however it is only evaluated on cases where these constraints were known beforehand. Therefore our paper specifically addresses the issue of the latter constraint, i.e. how to find an estimate for $a_{\min }$.

A common method to invert $\mathrm{RO}$ signals is phase matching (PM)[4]. The signal is integrated over time and transformed into a complex function of the impact parameter. The bending angle is derived by taking the derivative of the phase of this function. The significance of the its amplitude, however, is less clear. It can be used to determine the lower cut-off impact parameter of the data, and possibly to detect the presence of a SR layer.

In this paper, we investigate the amplitude of the PM with a focus on the relation between its decline and $a_{m i n}$. To do this we perform simulated occultations using realistic atmosphere data[5], and present results both for ideal conditions and for the case when instrument noise is added.

\section{REFRACTIVITY AND IMPACT PARAMETER AT GROUND}

Assuming spherical symmetry, the path of an optical ray passing through the atmosphere satisfies Bouger's rule[6]. This rule defines a constant $a$, called impact parameter for each ray:

$$
a=r n(r) \sin (\phi)
$$

where $r$ denotes the magnitude of a vector going from the center of curvature of the Earth to the position of the ray, $n(r)$ denotes the atmosphere's refractive index at the ray's position, and $\phi$ denotes the angle between this radius vector and the ray's direction vector. At a ray's lowest point $r_{t}$ (the tangent altitude), $\phi$ equals $\frac{\pi}{2}$ radians and (1) evaluates to:

$$
a=r_{t} n\left(r_{t}\right)
$$

In the case of an atmosphere with a SR layer, the inverse Abel transform introduces a bias in the refractivity profile, as shown in the left panel of Fig. 1. Below the upper boundary of the SR layer (around $1.2 \mathrm{~km}$ ) the true (solid line) and biased (dashed line) refractivities differ from each other. While the true refractivity has its lowest point at $h=0$, the biased curve terminates some 100 meters above ground. It is known that the impact height that corresponds to the lowest point on both 
these curves must be equal to the impact height at the lowest point on the bending angle curve (right) $\left(a_{m i n}\right)$. Since we know that the tangent point radius at this point is equal to the radius of curvature of the Earth, $r_{c}$, we can use (2) to directly calculate the refractive index at ground as $n\left(r_{c}\right)=\frac{a_{\min }}{r_{c}}$, and the refractivity is found through

$$
N=(n-1) \cdot 10^{6} \text {. }
$$

From accurate knowledge of $a_{\text {min }}$ we can therefore calculate $N\left(r_{c}\right)$ even in the case of SR. However, the bending angle curve in Fig. 1 is too ideal compared to a retrieved bending angle. It is created from a forward Abel transform of a refractive index profile, and takes no account of the wave properties of the signal. The lowest point in a bending angle diagram generated by PM on a real (or realistically simulated) signal has no well determined $a_{\text {min }}$. Thus, in order to use this method for calculating the lowest refractivity we need to develop a method to correctly determine $a_{\min }$, and in order to do that we need to make a close analysis of the structure of the simulated signal data close to ground.
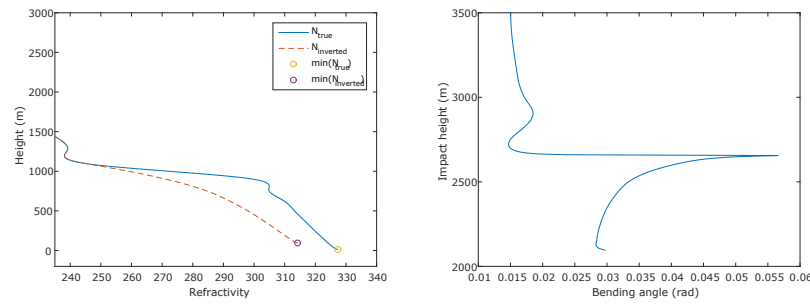

Fig. 1. Example of super-refractivity. The inverted refractivity profile (left) suffers from a bias. Both refractivity profiles yield the same bending angle (right).

\section{PHASE MATCHING}

For a signal $u(t)$ received in Low Earth Orbit (LEO), Jensen et al. define the PM operation as follows[4]:

$$
U(a)=\int_{t_{\min }}^{t_{\max }} u(t) \exp \left(-i k_{0} s(t, a)\right) d t
$$

where $s(t, a)$ is the optical path length of a model ray path. The function $s(t, a)$ can be defined for any $a$, and as such a range is chosen to include the Earth's surface by a large margin. The phase of $U(a)$ is then differentiated with respect to $a$ to yield the bending angle $\alpha(a)$. Consequently, $\alpha(a)$ is defined for values that do not make physical sense, and we look into $|U(a)|$ to determine the "cutoff point", $a_{\text {min }}$. We define $\hat{U}(a)$ as the normalized $U(a)$ with respect to its values at high altitudes. The $|\hat{U}|$ function has a very clear structure; all instances will have a sharp decline as the transmitter is shadowed by the Earth at low impact heights. Since $a_{\text {min }}$ has to be located above this decline, we define a halfway point $a_{1 / 2}$ such that $\left|\hat{U}\left(a_{1 / 2}\right)\right|=\frac{1}{2}$. An example of a typical $|\hat{U}|$ function can be seen in Fig. 2, with $a_{\min }$ and $a_{1 / 2}$ highlighted. From these quantities we also define $\Delta a=a_{\min }-a_{1 / 2}$.

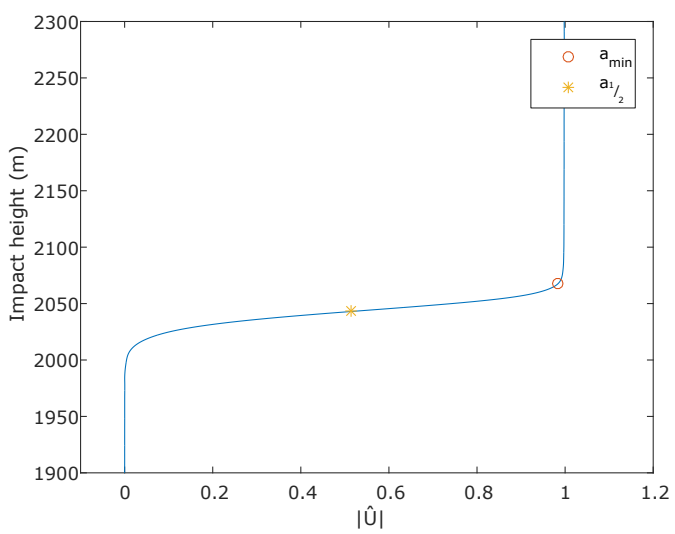

Fig. 2. $|\hat{U}(a)|$ for case 1 from the reference dataset[5]. The impact parameter corresponding to the Earth's surface is located just above the sharp decrease of amplitude.

\section{SIMULATIONS}

In order to simulate a wave passing through the atmosphere we have implemented a wave optics propagator (WOP)[7], inspired specifically by the work of Rasch[8]. The WOP solves the Helmholtz equation iteratively along an axis in a twodimensional space. This technique has been called the multiple phase-screen technique[9]. We model the Earth by multiplying the wave amplitude with a tapering window if $r>r_{c}$ :

$$
w(r)=e^{-\frac{\left(r-r_{c}\right)^{2}}{L_{T}^{2}}},
$$

where $r$ denotes the distance from the center of curvature, and $r_{c}$ denotes the Earth's radius of curvature. We use a tapering length $L_{T}=100 \mathrm{~m}$. The purpose of this window is to prevent artifacts that occur if the wave is not continuous. As input the WOP needs a one-dimensional atmosphere profile of refractivity, which is then assumed to be a spherically symmetrical atmosphere of the Earth. The output of the WOP is the amplitude and phase of the signal received in LEO, along with the corresponding geometry of transmitter, receiver and Earth.

We use the 55 reference cases defined by Healy[5] as input to the WOP. These cases are divided into 4 categories, where each category is increasingly "difficult" to invert. Category 4 cases have such a large refractivity gradient that they are SR, and ducting occurs. After the WOP we perform PM on the simulated signals and note their respective $\Delta a$ values. 


\section{NOISE AND FILTERING}

In order to make the signals more realistic we add noise to the WOP output. As the signal used in the simulation was of L1 frequency, and the amplitude was normalized to 1 at high altitude, an appropriate (complex-valued) Gaussian noise was added with $\sigma=0.0537$, which corresponds to a signal-tonoise ratio of $25 \mathrm{~dB}$ at high altitude. As the sample rate of the signal is $100 \mathrm{~Hz}$, this represents a signal-to-noise power density of $45 \mathrm{dBHz}$, which is a pessimistic value in comparison to the $55 \mathrm{dBHz}$ expected for current and future RO instruments[10]. To filter the noise, we employed a sliding window in which we use linear regression to fit a polynomial of the second degree to each point in the noisy amplitude function. For this study, we use a window length of $170 \mathrm{~m}$ to remove all jaggedness from the decline of $|\hat{U}|$. In the left panel of Fig. 3 is an example of a noisy profile, and to the right, how it looks after filtering.
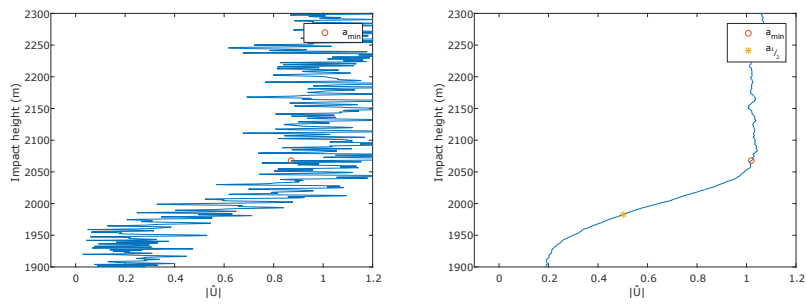

Fig. 3. $|\hat{U}|$ function with noise (left), and filtered using a sliding polynomial fitting window (right).

\section{RESULTS}

In Fig. 4 and Fig. 5 we show the $\Delta a$ values determined with ideal conditions and noisy conditions. We present the mean $(\mu)$ and standard deviation $(\sigma)$ of the distributions as well. The super-refractive (category 4) profiles in the dataset are highlighted with circles. Figures 6 and 7 show the resulting errors in refractivity if $N$ is calculated using (2) and (3), with $a_{\min }$ approximated using $a_{1 / 2}$ and the mean of $\Delta a$, i.e.

$$
N_{\text {est }}=10^{6}\left(\frac{a_{1 / 2}+\mu(\Delta a)}{r_{c}}-1\right)
$$

The errors are relative and are calculated by $N_{\text {error }}=$ $\frac{N_{\min }-N_{\text {est }}}{N_{\min }}$.

It is clear that the SR cases are clustered around $\pm 20^{\circ}$, which would correspond to the moist, subtropical regions where these ducting type of profiles are quite common. It is also clear that there appears to be no particular difference in the accuracy of the estimates for the SR cases as compared to the entire set, but they seem to be more precise. There is no particular reason as to why this would be the case, and it is most likely caused by the low number of profiles used in the analysis. The small difference between the results of noise-free and noisy signals shows that the method is robust to realistic instrument noise.

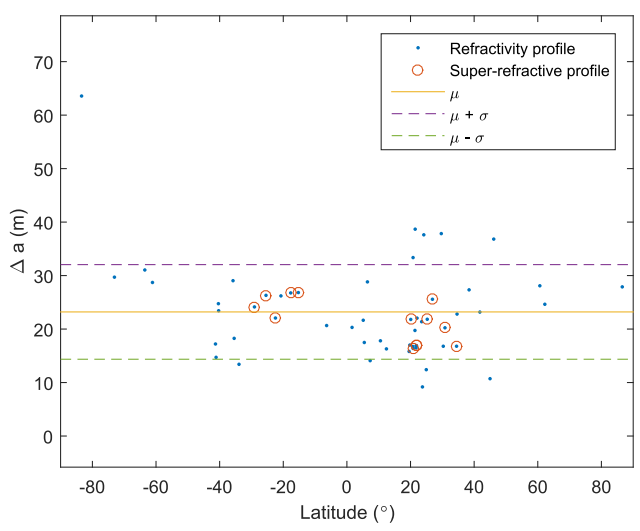

Fig. 4. The spread of $\Delta a$ values from noise-free signals by latitude, with category 4 cases highlighted.

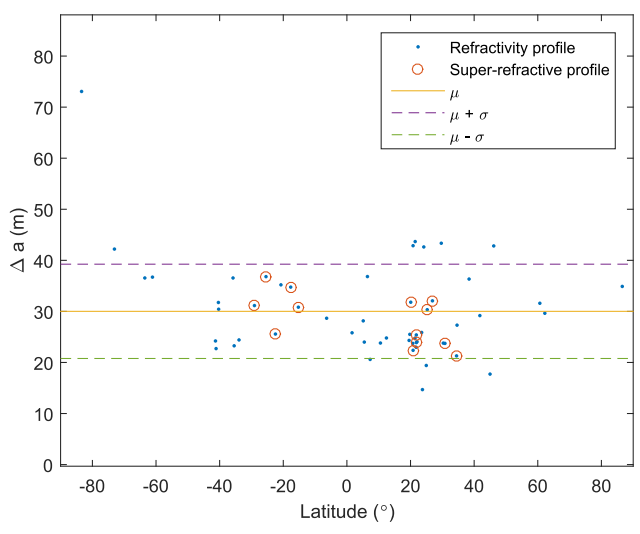

Fig. 5. The spread of $\Delta a$ values from noisy signals by latitude, with category 4 cases highlighted.

\section{DISCUSSION AND CONCLUSION}

The results in this paper indicate that refractivity at the bottom of the atmosphere can potentially be determined from the amplitude of the PM output. However, there are several limitations and challenges that need to be addressed before this method is ready for real occultation data.

The proposed method relies on a certain type of boundary condition for the Earth. The one used in these simulations is not chosen because it is accurate in a physical sense. Further simulations with more accurate boundary conditions might be needed.

The surface of the Earth is not entirely smooth, and as such finding an accurate value for $a_{\min }$ in cases of varying 


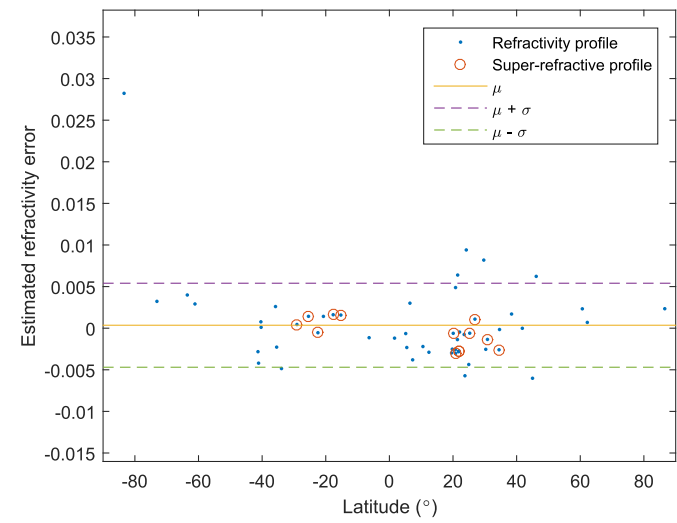

Fig. 6. The spread of relative $N$ errors from noise-free signals by latitude, with category 4 cases highlighted.

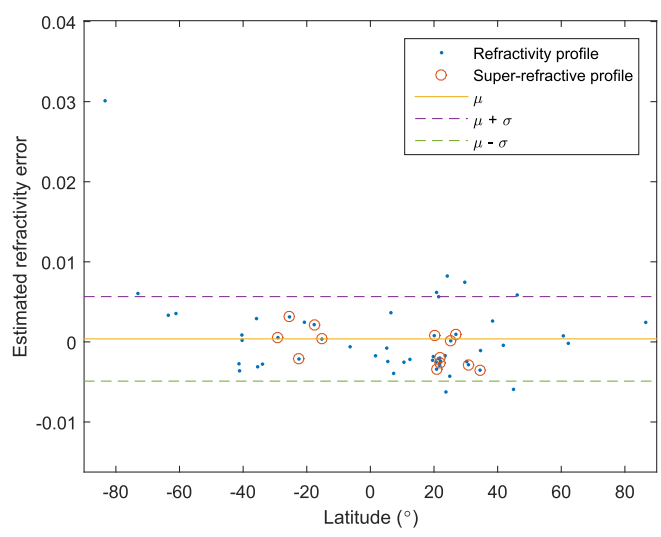

Fig. 7. The spread of relative $N$ errors from noisy signals by latitude, with category 4 cases highlighted.

topography seems unrealistic. Regions where the terrain is flat (e.g. the oceans) seem more suitable.

We used our proposed method to find $N_{\min }$ on the same data where we calculated the mean $a_{\min }$. This biases the results, and independent data is needed to make a proper validation. This can be achieved using in-orbit RO measurements and corresponding refractivity data, which is our planned next step for further investigations.

To conclude, we show that a simulated RO signal transformed by the PM integral contains some information about the lowest possible impact height. The fact that SR cases do not exhibit any form of bias implies that these results have the potential to aid in determining one of the constraints needed for the reconstruction method proposed by Xie et al. Furthermore our results indicate that the performance of our method is not significantly affected by noise. How this approach could be applied to real RO data is a question which requires in-depth analysis. We hope to be able to address this question in our future research.

\section{ACKNOWLEDGEMENTS}

The authors would like to thank The Swedish National Space Board and The Knowledge Foundation (KKS) for the financial support of this research project.

\section{REFERENCES}

[1] E. Robert Kursinski, George A. Hajj, Stephen S. Leroy, and Benjamin Herman, "The gps radio occultation technique," Terrestrial, Atmospheric and Oceanic Sciences, vol. 11, no. 1, pp. 53-114, 2000.

[2] Sergey Sokolovskiy, "Effect of superrefraction on inversions of radio occultation signals in the lower troposphere," Radio Science, vol. 38, no. 3, pp. 1058-24/14, 2003.

[3] Feiqin Xie, Stig Syndergaard, E. R. Kursinski, and Benjamin M. Herman, "An approach for retrieving marine boundary layer refractivity from gps occultation data in the presence of superrefraction," Journal of Atmospheric and Oceanic Technology, vol. 23, no. 12, pp. 1629-1644, 2006.

[4] Arne S. Jensen, Martin S. Lohmann, Alan S. Nielsen, and Hans-Henrik Benzon, "Geometrical optics phase matching of radio occultation signals," Radio Science, vol. 39, no. 3, pp. RS3009-RS3009, 2004.

[5] Sean B. Healy, "Optimizing tracking strategies for radio occultation. task 1 - the profile dataset," Tech. Rep., ECMWF/EUMETSAT, 2012.

[6] Max Born and Emil Wolf, Principles of Optics: Electromagnetic Theory of Propagation, Interference and Diffraction of Light, Pergamon Press, 1964.

[7] H. H. Benzon, A. S. Nielsen, and L. Olsen, "An atmospheric wave optics propagator - theory and application," Scientific report 03-01, Danish Meteorological Institute, 2003.

[8] Joel Rasch, "Theory and implementation of an end-toend radio occultation simulator," Technical Report 10, Earth and Space Sciences, Chalmers University of Technology, Göteborg, Sweden, 2014.

[9] D. L. Knepp, "Multiple phase-screen calculation of the temporal behavior of stochastic waves," Proceedings of the IEEE, vol. 71, no. 6, pp. 722-737, 1983.

[10] Magnus Bonnedal, Jacob Christensen, Anders Carlström, and Anders Berg, "Metop-gras in-orbit instrument performance," GPS solutions, vol. 14, no. 1, pp. 109-120, 2010. 